\title{
Opportunities for UAV mapping to support unplanned settlement upgrading
}

\author{
C. Gevaert ${ }^{1}$, R. Sliuzas ${ }^{2}$, C. Persello ${ }^{3}$, G. Vosselman ${ }^{4}$ \\ ${ }^{1234}$ University of Twente/Faculty ITC, Enschede, the Netherlands \\ c.m.gevaert@utwente.nl,r.sliuzas@utwente.nl, c.persello@utwente.nl, george.vosselman@utwente.nl
}

\begin{abstract}
The effort to improve sub-standard living conditions in unplanned settlements is often hindered due to a lack of adequate spatial information describing the baseline situation and changes occurring during and after the upgrading process. Low-cost Unmanned Aerial Vehicles (UAVs) could provide very detailed, up-to-date spatial information for small unplanned areas as and when required. To investigate the utility of such platforms in settlement upgrading, UAV flights were conducted over approximately 150 ha of unplanned settlements in the City of Kigali in May and June 2015. Data obtained with UAV were supplemented by an analysis of the spatial information needs of various stakeholders involved in the upgrading project. In the context of the upgrading project, the results of this study identify four main benefits of using UAV imagery. First, it could replace the $200825 \mathrm{~cm}$ ortho-imagery by up-to-date $3 \mathrm{~cm}$ imagery in current workflows for map updating. Second, it enables the extraction of additional information which was previously unavailable, such as detailed elevation data to support surface water runoff analysis and drainage capacity calculations. Third, it speeds up field work and fourth, the imagery provides a foundation for communication between different stakeholders.

When using UAVs it is also important to take many practical considerations into account, as well as the societal and ethical context. Results of the first experiences in Kigali indicate that while the use of UAV is not generally perceived as a problem by the local population, fear of forced displacement and expropriation may raise concerns amongst the residents. Communication between the UAV operator, the local government, local leaders and with the residents before and during flights, and sharing the benefits of the acquired information are important to mitigate these fears. Moreover, the resolution and quality of the images is such
\end{abstract}


that privacy concerns and issues such as their potential to be used to the detriment of residents of such areas should not be ignored.

Keywords: Unmanned Aerial Vehicles, unplanned settlements, housing upgrading projects, spatial information requirements.

\section{I.Introduction}

Spatial data is considered essential for unplanned settlement upgrading projects (Kohli et al., 2013, Abbott, 2002; Taubenböck and Kraff, 2013). Obtaining an accurate base map of these areas provides a sound basis for designing technical interventions (Paar and Rekittke, 2011; UN-Habitat, 2012), as well as improving the communication between stakeholders (Barry and Rüther, 2005), and empowering local authorities and communities (Abbott, 2003).

Remotely sensed imagery is a key source of spatial information, as it can provide an objective, up-to-date overview of the physical situation in the settlement (Taubenböck and Kraff, 2013). Mason and Fraser (1998) identified seven important roles of satellite imagery for unplanned settlement management: identification of unplanned settlements, identifying changes in the boundaries of these settlements over time, generation of surface data, land use classification, extraction of buildings and other objects for mapping purposes, detailed settlement mapping for spatial modelling purposes, and reconnaissance. However, small buildings and narrow footpaths characteristic of unplanned settlements may hinder the interpretation of commercial satellite imagery with half-meter resolution (e.g. Kuffer et al., 2014).

Unmanned Aerial Systems (UAVs) or Remotely Piloted Aircraft Systems (RPAS) also known as drones, are defined as small aircraft operated without an onboard pilot (Nex and Remondino, 2014). Similar to traditional aerial image acquisition, a UAV is mounted with a camera which takes images of the study area as it flies over. The individual images can be stitched together to create a 3D model in the form of a point cloud, as well as obtain a high-resolution Digital Surface Model (DSM) and orthomosaic. The orthomosaics obtained from the UAV imagery can reach a resolution of a few centimeters (Nebiker et al., 2008). Although this is similar to the resolution which can be obtained by aerial photography, UAVs cost less and, at least for 
relatively small areas, are more flexible in acquiring data (Nex and Remondino, 2014). As such, this possibility of obtaining high-resolution spatial data in a relatively cheap and dynamic manner could be a practical approach to provide essential baseline information in unplanned settlement upgrading projects.

However, for a UAV to be useful for unplanned settlement mapping, the workflow must not only meet the technical requirements of the user, but the new technology must fit into the local context (Pannell et al., 2011) and its use should be ethical and provide adequate protection of the privacy of those whose properties and even their bodies, are recorded on the images. It is therefore important to analyze the spatial information requirements of the potential end-users as well as the social context. The main users of UAVs for unplanned settlement mapping are likely to be governmental institutions or organizations operating on their behalf as partner or consultant. Unfortunately, studies from cities in six different developing countries indicated that data sharing is limited as spatial data collection and products are often restrained to experts in the governing body and private sectors (Baud et al., 2014). Dependency on spatial technology may therefore increase social exclusion (Pfeffer et al., 2013). It is important to analyze how the benefits of the UAV imagery may not only serve the governing bodies, but also how they may be distributed amongst stakeholders, in particular the local population as in the case of informal settlements they tend to be the most disadvantaged and vulnerable members of society

The process of using UAV for mapping purposes has been well documented (e.g. Nex and Remondino, 2014; Colomina and Molina, 2014). In general terms, the UAV information acquisition workflow consists of: (i) flight planning and execution, (ii) ground control point (GCP) acquisition, and (iii) data processing. The flight planning consists of, for example, selecting the UAV platform and defining the flight parameters. Depending on the available budget, project area and required image resolution, a suitable UAV platform and payload can be selected. For example, a fixed wing (airplane-like) UAV may cover a larger area with one flight, but requires an extended take-off and landing zone. On the other hand, rotary wing systems which are powered by vertically oriented propellers, or rotors, require more battery power which reduces their flight time. However, rotary-wing UAVs are capable of taking off and landing vertically, and are more agile in their image acquisition. Some UAVs have a fixed payload (such as a built-in camera), whereas others allow the user to 
change the payload, thus giving the user more control of the spectral or spatial resolution of the acquired data products. After the platform is selected, the flight path must be defined. Many consumer-grade UAVs are capable of automatic flight planning, where a grid pattern is automatically generated from the flight height and overlap defined by the user. The flight height and payload will influence the spatial resolution of the output orthomosaic, whereas the overlap influences the quality of the data products. Finally, before executing flights one must first check the UAV flight regulations. In Rwanda, flight permission is now regulated by the Rwanda Civil Aviation Authority (RCAA) through the ministral regulations $\mathrm{N}^{\circ} 01 / \mathrm{MOS} / \mathrm{Trans} / 016$ of 26/04/2016 relating to unmanned civil aircraft systems, however, these were not in place during the mission described here.

Furthermore, most UAV systems require GCPs to improve and verify the geometric accuracy of the orthomosaic, DSM, and point cloud obtained from the UAV images. The precision of the GPS utilized must conform to the high spatial resolution of the UAV data products. The GCPs may be collected during the UAV flights by placing targets on the ground before the flights so they are visible in the UAV images, and measuring their coordinates. Alternatively, GCPs can be added afterwards by obtaining the coordinates of permanent structures which can also be identified in the UAV imagery.

Finally, the data processing stage converts the raw images into useable data products. The theoretical background of this process is described by Hartley and Zisserman (2003), whereas a good practical explanation is given by Nex and Remondino (2014). First, a unique descriptor is used to identify pixels from various images which represent the same object. After these so-called tie points are listed, the camera calibration and image orientation are performed through a bundle block adjustment. This identification of the interior and exterior image parameters is also known as "structure from motion". In this step, the GCPs can be added to improve the geometrical accuracy and verify the quality of the parameter estimations. The sparse 3D point cloud obtained from this step is then enriched through dense matching algorithms (Remondino et al., 2014). Next, a DSM is interpolated from this point cloud. The original images are stitched together, using the elevation information from the DSM to form an orthomosaic. Various photogrammetric software are available which can execute the process from images to dense point cloud, DSM, and 
orthomosaic semi-automatically. Comparisons of such software are provided by e.g. Remondino et al. (2014) and Sona et al. (2014).

The objective of this paper is to analyze the potential of UAVs to support unplanned settlement upgrading projects, to describe the first perceptions of various stakeholders, to identify important factors which should be taken into account for the diffusion of this technology and its benefits amongst stakeholders, and to underline the ethical concerns of privacy and possible misuse of the obtained information. The paper is based on the results of UAV image acquisition in the context of upgrading projects in the City of Kigali, Rwanda in May/June, 2015. However, it should be noted that this study is more a reflection on the process of obtaining and using UAV images in the context of urban upgrading rather than a technical study of image quality or application.

The materials and methods section provides an overview of the context of the case study and the UAV flights conducted over the area. In the results section, the spatial data requirements of upgrading projects are analyzed, and the added value of using UAV data is described through the identification of four key benefits. The practical considerations and social and ethical context are also described in the results. Finally, the discussion of the results leads to the identification of key factors which should be taken into account to support the diffusion of UAVs and their related information extraction techniques, for unplanned settlement upgrading.

\section{II.Material and methods}

In May 2015, a number of UAV flights were conducted in Kigali by the University of Twente - Faculty ITC with the support of Affordable Housing Unit of the City of Kigali One Stop Center (CoK-OSC), the Rwanda Civil Aviation Authority (RCAA), and village representatives. Eighty-nine flights were made in total, taking more than 15,000 images to cover approximately 150 ha of unplanned settlements (Figure 1). The main study area covers parts of Agatare, Rwampara, Biryogo and Kiyovu cells of the Nyarugenge District in the 
city of Kigali, which was selected by the Rwandan government for an urban upgrading project. The Nyarugenge project area is roughly 86 ha in size, with an estimated 3,977 households and 18,914 individuals (GISTech Consultants LTD, 2015). The houses are generally made of mud and wood with corrugated iron roofs. A few localized improvements have previously been made regarding access and drainage, but the current project aims to provide a more comprehensive improvement. This area was selected to be a pilot project which aims to not only identify infrastructure improvements in the area, but also to develop successful strategies for participatory engagement and designing slum upgrading projects in Kigali and the secondary cities of Rwanda. The project targets issues including: storm water drainage, sewerage, drinking water supply, roads, electricity and public lighting, and housing improvement. The project started on in December 2014 and the intervention design and cost plans were provided at the end of June, 2015. As of November 2015, activities in the project area include environmental and social safeguards reporting and a detailed design of the prioritized interventions.

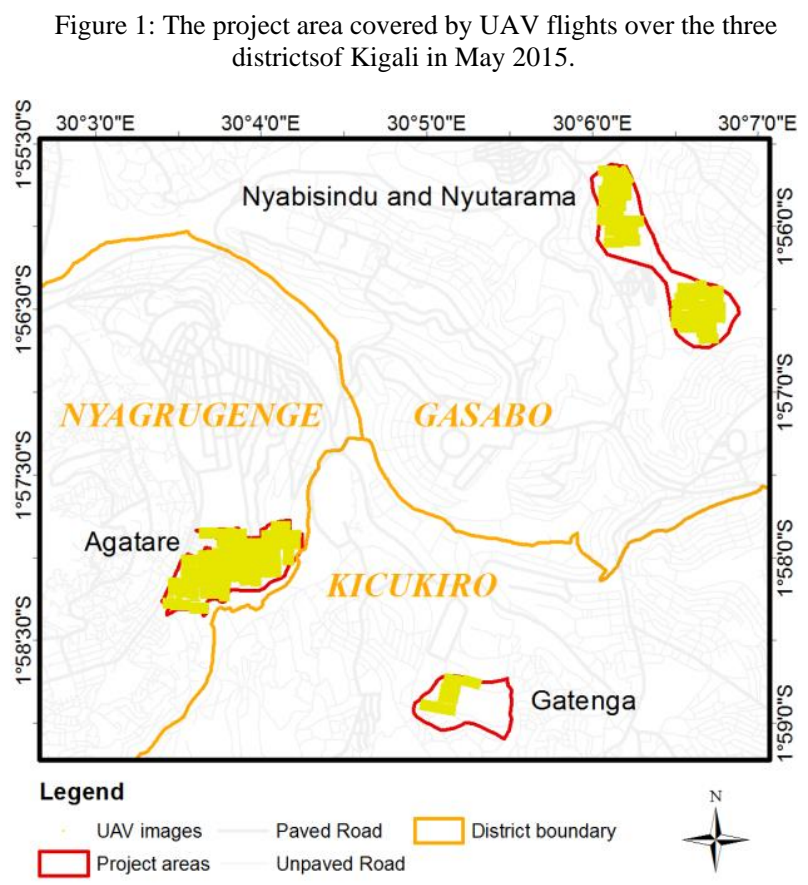

The UAV utilized for the flights was a DJI Phantom 2 Vision+ quadcopter (i.e. a rotary-wing UAV with four rotors) with a 14 Megapixel RGB camera with a fish-eye lens $\left(\mathrm{FOV}=110^{\circ}\right)$. Examples of some derived products, the dense point cloud and ortho-image, are given in Figures 2 and 3 respectively. The UAV 
orthomosaics were provided to the CoK-OSC and were used by the consultants responsible for the environmental and social impact assessments and the consultants responsible for the detailed infrastructure planning. The orthomosaics were also made available at village level offices where they could be viewed and used by citizens engaged in public participation processes. This provides the opportunity to observe how the UAV images are actually used by various project stakeholders.

Interviews were also conducted with both institutional and local stakeholders to identify important spatial information requirements for the upgrading projects, the perceived use of UAV for these activities, and to assess the attitudes of the local population towards the usage of UAV.

Figure 2: Sample of the ortho-image obtained from the UAV data over the project area.

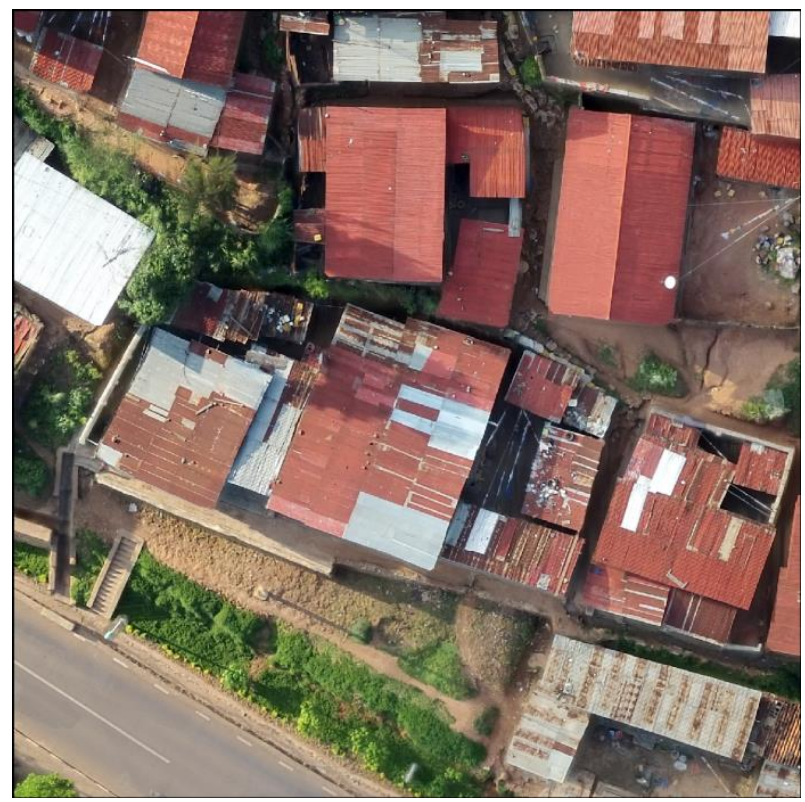

Figure 3: Sample of the 3D model (mesh) obtained from the UAV data over the project area.

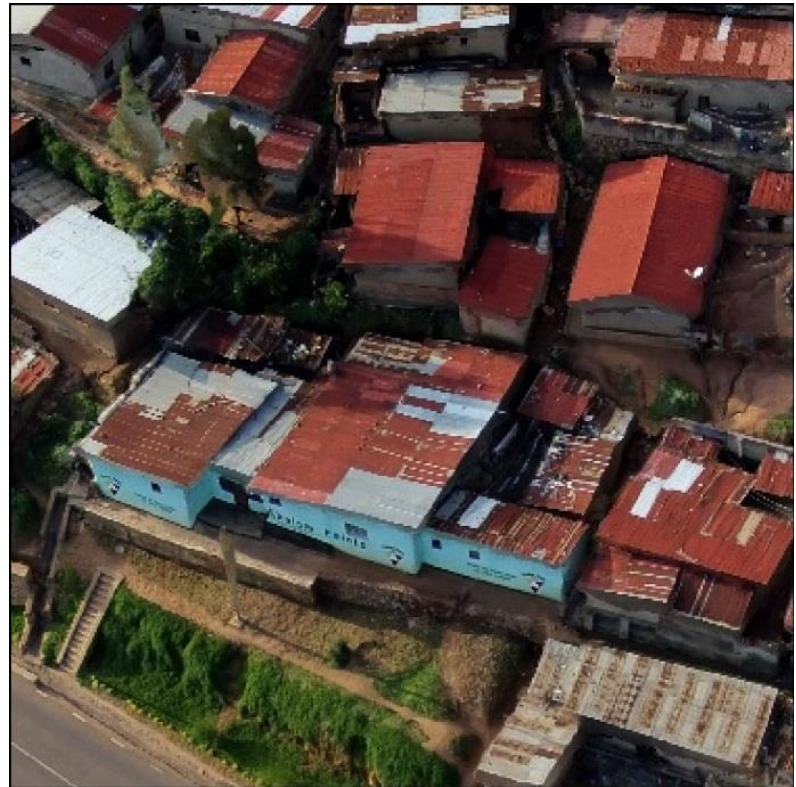

\section{III.Results}

\subsection{Spatial information requirements for upgrading projects}

An upgrading project consists of a number of phases: feasibility studies, detailed studies, developing project options, detailed design and project implementation (Davidson and Payne, 1983). The first two stages require information which describes the project area on four aspects: population and housing needs, the conditions of the project site, assessment of the current site development, and finally the institutional and financial 
framework. Although much of the socio-economic information required for upgrading projects may be stored in GIS databases, remotely sensed data such as airborne imagery or UAVs are limited to describing the physical characteristics of the settlement. We therefore restrain our analysis to the physical spatial information requirements which excludes the information regarding population and housing needs and the institutional and financial frameworks. However, remotely sensed data can provide enormous benefits in describing the physical characteristics of the project site environmental conditions (i.e. topography, vegetation, presence of hazards) and especially in describing the existing site development. The latter includes characterizing the existing buildings, house layouts, plot coverage, land use, and accessibility.

As the Nyarugenge upgrading project is intended to be a pilot project, there was not a well-established methodology regarding spatial data norms for upgrading projects in the City of Kigali. The spatial data requirements were therefore not clearly defined at the start of the feasibility and detailed design project which was completed in July 2015. Rather, they were based on the recommendations of a World Bank consultant (Banes, 2015), and were further specified in an interactive manner during the project execution by the contracting authorities (Rwanda Housing Authority, CoK-OSC, and Nyarugenge District) and consultants.

Table 1 lists the spatial data collected by the consultants, which could be equated to the current spatial data requirements. Initially the spatial information collected for the project was either: (i) provided by authorities, (ii) digitized from the 2008 ortho-imagery, or (iii) collected in field with a GPS. The collection of the spatial information was supported by the Rapid Planning Consortium, an action-oriented research project supported by the German Federal Ministry for Education and Research and UN-Habitat to develop a trans-sectoral urban infrastructure planning methodology, for which Kigali is one of the three case cities (Rapid Planning Consortium, 2015). 
TABLE 1: SPATIAL INFORMATION COLLECTED BY GIS CONSULTANTS FOR THE NYARUGENGE DISTRICT UPGRADING PROJECT

\begin{tabular}{ll}
\hline \hline \multicolumn{1}{c}{ Spatial Layer } & \multicolumn{1}{c}{ Source } \\
\hline Aerial imagery & $25 \mathrm{~cm}$ true-colour orthophoto from 2008 \\
Satellite imagery & $50 \mathrm{~cm}$ Pléiades imagery from 2014 (Rapid Planning \\
& Consortium) \\
DTM points at $10 \mathrm{~m}$ intervals provided by Rwanda & Natural Resources Authority (RNRA) \\
Building Footprints & Footprints were digitized from satellite imagery and \\
& roof material was obtained by sampled questionnaires \\
& with the support of Rapid Planning, \\
Roads (type and material) & Digitized from satellite imagery, GPS in field \\
Drainage & Digitized from satellite imagery, GPS in field \\
Power lines & Provided by the power company, GPS in field \\
Water pipelines & Provided by the water utility company \\
Land use & Digitized from aerial photos, field survey \\
Services & GPS in field \\
Parcels & Provided by RNA \\
Administrative boundaries & Provided by National Institute of Statistics Rwanda \\
& (NISR) \\
\hline \hline
\end{tabular}

\subsection{Added value of UAV imagery to provide the required spatial information}

Previously, upgrading projects relied on the availability of satellite or airborne imagery. The benefits of UAV imagery as opposed to conventional imagery can be categorized into four main aspects: (1) by providing more accurate information through direct replacement of conventional imagery in existing workflows, (2) by providing additional, previously inaccessible, information, (3) by reducing field work, and (4) by providing a basis for communication amongst stakeholders.

Regarding the first aspect, the imagery obtained from UAVs could be directly integrated by replacing satellite imagery in the existing spatial data workflows of the upgrading project. However, as opposed to airborne or satellite imagery, UAVs are a more flexible information acquisition platform. This potentially allows data to be collected more frequently, whether it's one large survey before a project starts or incremental surveys to observe and map changes during a project implementation phased over many years. Apart from the ability of providing up-to-date information, the spatial resolution of the UAV imagery is significantly better than that of conventional airborne imagery. Through direct replacement, rather than for example relying on an ortho-photo from 2008 with a $25 \mathrm{~cm}$ resolution, an up-to-date orthomosaic with a resolution of $3 \mathrm{~cm}$ could 
be used to digitize the objects of interest. This is apparent in Figure 4, which displays a part of the project area in the 2008 orthophoto (left) versus the UAV orthophoto (right). Notice how: buildings and other objects are more clearly visible in the UAV orthophoto, which facilitates digitization; the appearance of new buildings or improvements to roofs, which increases the accuracy of the digitized information.

Figure 4: The added value of the UAV data is clearly visible when comparing the information provided by the 2008 orthomosaic (left) to the 2015 UAV orthomosaic (right). Note the enhanced visibility of objects in the scene as well as the appearance of new structures.
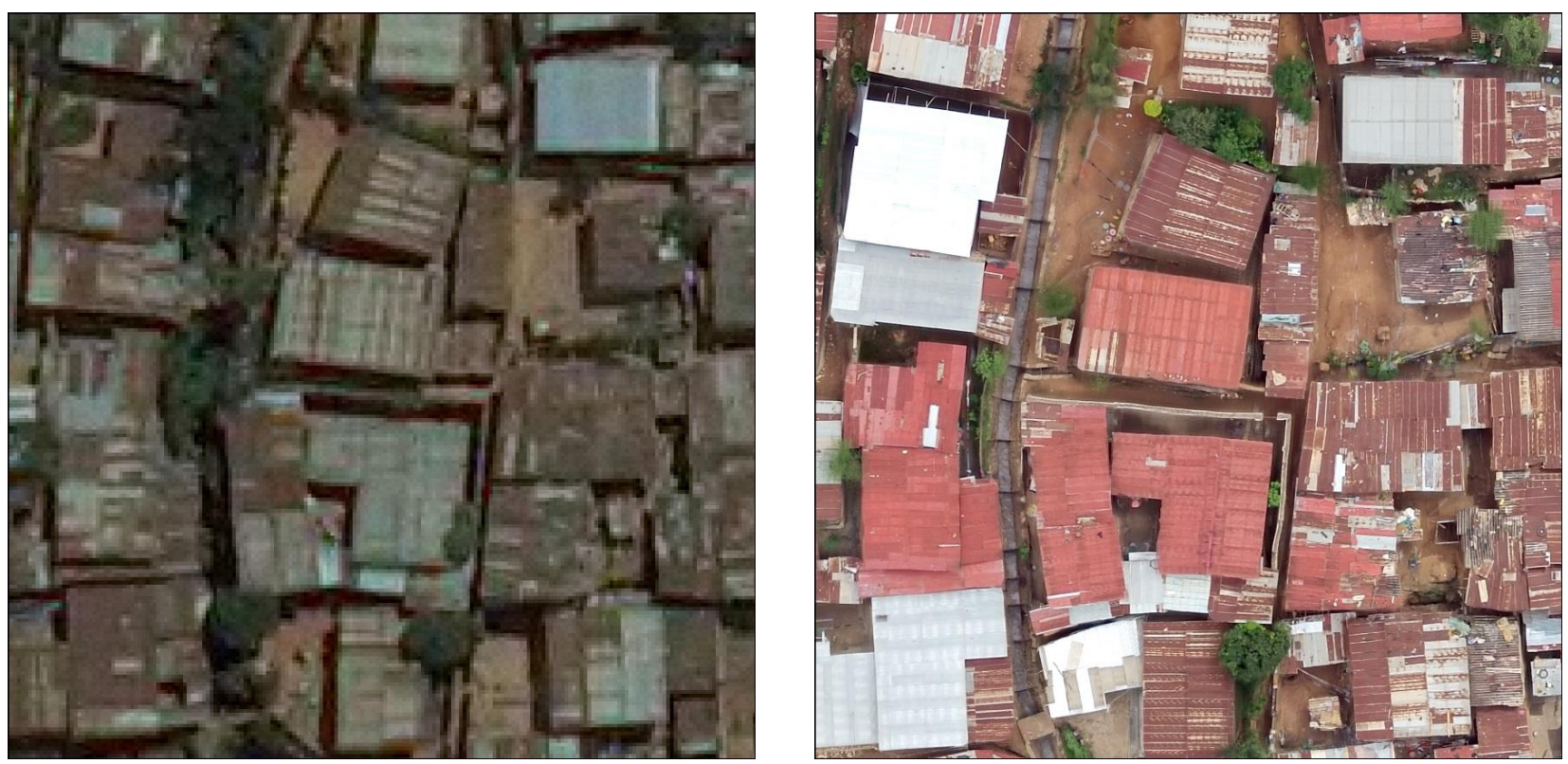

The second main benefit is the provision of additional information. The higher spatial resolution of the orthomosaic as opposed to the aerial imagery allows for new objects of interest to be identified, such as lamp posts which indicate the presence of street lighting and the waste accumulation areas, which can be used to effectively plan waste collection points. Furthermore, more detailed attribute data of the existing objects can be obtained. For example, the roof material and condition of individual buildings can be observed and quantified. Such information may be used to monitor the implementation of the KCMP as well as provide baseline statistics describing the general conditions within the settlement. The 3D data obtained by the UAV provides information regarding building height and the local topography. Especially the latter may support the detailed design of upgrading interventions. For example, the drainage in the Agatare project area was mapped by GPS points in-field and measuring the width of the drain at regular intervals. Using the Digital Terrain Model (DTM) extracted from UAV imagery, the drainage capacity could be calculated more 
accurately (though still concealed in some areas by trees etc.), facilitating the design of more adequate interventions. In situations where the terrain is not visible due to concealment by trees or other structures (i.e. occlusions), terrestrial imagery may be integrated with the UAV imagery to provide a more complete 3D input for drainage models, similar to the work of Meesuk et al. (2015).

The ability to provide up-to-date imagery and to identify additional objects and attributes was perceived by the consultants to be a large benefit for the existing workflow by reducing the time and cost of subsequent field verification. This is the third main advantage of the utilization of UAVs, namely the reduction of field work. On the one hand, the ability to obtain more accurate and detailed information reduces the amount of data which must be collected or corrected in the field. On the other hand, as the project members have access to an up-to-date map, it is easier to plan their field work as the location of and accessibility to certain objects of interest are clearly visible. However, the reduction of the time required for the field work should be weighed against the time required to acquire and process the UAV imagery.

Finally, the high detail of the data products provides an intuitive environment which facilitates discussions between stakeholders. The UAV orthomosaics, overlaid with the designated project interventions, were presented by project members to the sector offices during the monthly community meeting in October 2015. Project members mentioned that, due to the detail, many residents could recognize their houses and other landmarks. This helped them locate the proposed interventions, thus providing a foundation for discussions between stakeholders. Although the upgrading project aims to limit expropriation, the introduction of utilities and improvement of infrastructure will require extra space and almost certainly results in some expropriation. As the spatial layout of buildings is more clearly visible in the UAV orthomosaic, planners are better able to position infrastructure to minimize demolition, explain why interventions are designed in certain positions, and as a result why specific structures are selected for relocation. The printed maps were left at the sector offices, and hardcopies of the orthomosaics are therefore directly available to the local population. This is a simple way through which the residents could also benefit from the UAV imagery. Further analysis should identify additional uses of the imagery by the local citizens and as well identify concerns of an ethical nature related to mission planning, the mapping process, data ownership, data use, marginalization etc. (Rambaldi et 
al., 2006) that may or have been recognized as problematic or potentially so.

\subsection{Issues identified as potential bottlenecks regarding the use of UAV}

\section{A. Practical considerations}

Although from a scientific perspective, UAVs appear to be a promising method for spatial data collection, there are also practical considerations regarding the technological limitations of UAV platforms, data processing, and specialized knowledge. The technological characteristics of the UAV platform also affect the utility of UAVs as a data acquisition tool. Firstly, imagery cannot be acquired during rainy or windy weather conditions. Secondly, the range of the platform limits the extent of area covered per flight, and requires the take-off location to be close to the flight area. This may be difficult in unplanned settlements, where dense construction, narrow footpaths and overhanging power lines or vegetation make it difficult to find adequate take-off locations and which may slow down data acquisition.

Back in the office, there are also practical challenges to obtain the required information from the raw UAV images. First, from a data quality perspective, a high image overlap, or redundancy, increases the quality of the 3D model. However, the large number of images may also incur data storage problems (Baiocchi et al., 2014). Furthermore, processing the imagery currently requires specialized software and advanced hardware requirements. In recognition of the data processing bottleneck, the University of Twente - Faculty ITC, the Institut National de l'Information Géographique et Forestière (France), and The Netherlands eScience Center have initiated a project aimed at speeding up the open source photogrammetric processing software MicMac (NLeSC, 2015). This project resulted in a number of extensions to MicMac to speed up data processing through distributed computing and reduces memory requirements through a more efficient calculation of tie points. Such developments speed up data processing within a software environment which does not have licensing fees as well as reduce hardware requirements.

As with any new technology, there are costs associated with the initial adoption of UAVs for upgrading projects. Although the UAV itself may be relatively inexpensive, there are additional expenses regarding obtaining the proper permissions and documentation, training the pilots, and training the GIS specialists and 
photogrammetrists who must then process the data. However, it should be noted that once the UAV imagery is collected for an upgrading project, there are many other sectors which may also benefit from the obtained data products. Examples include: updating existing (smaller scale or outdated) topographic map data, supporting cadaster, valuation for tax collection purposes, and inspection for the monitoring of illegal construction.

\section{B. Social considerations}

Such practical issues of technological limitations, data processing, and investment costs may be overcome. However, they overlook one of the most important stakeholders involved in UAV data acquisition - the inhabitants of the settlements being flown over and their privacy. Based on the experiences of flying the UAV in Kigali, the first observation was the interest of the inhabitants during the flights. Many people were curious, crowding around to watch the flights, taking pictures and asking questions. However, a number of people were also concerned. Most concerns were based on the fear that the UAV was being used to survey the area and plan for expropriation. In this particular project, there was a very limited time to execute the UAV flights after permission was obtained. There was therefore insufficient time to fully address such community concerns through proper planning and awareness raising (Rambaldi et al., 2006). However, an effort was made to mitigate their fears by answering questions and explaining the purpose of the activities during the flight acquisition. Furthermore, the village leaders were notified about the flight activities beforehand so they could communicate this to the local population and mitigate concerns.

The utility of UAV data products, in the form of printed images, for the local residents was also analyzed through interviews. When shown some sample UAV images, a number of residents 'recognized' the images from previous land administration activities or the activities of the consultants involved in the Agatare project. Others indicated that it was very important to provide the users with some kind of training or explanation regarding how to interpret the images.

According to interviews with residents, the images were mainly considered to be useful for the village leaders. The general population mentioned uses such as giving a friend directions how to find their house, or 
as a memory to show their grandchildren how the neighborhood used to look like. Others mentioned that you could compare your house to your neighbor's house. This was also the most recurring theme in the utility of the images for village leaders. The UAV imagery was considered to be useful to identify which aspects (e.g. house typology) needed to be changed in order to comply with the KCMP. Village leaders also mentioned that they could use the images to help explain the government plans to the population. These observations hint at the strength of the top down influence of the government in Rwanda, and to the extent to which plans such as the KCMP are communicated to the local leaders. As a hardcopy of the images is now available at the sector offices, future analysis may identify additional uses of the images by the local officials and citizens.

\section{IV.Discussion}

Although there is a great enthusiasm regarding the detail of the imagery obtained from a UAV, practical aspects must be taken into account to determine which applications could maximally benefit from the UAV imagery. In the case of upgrading projects, the size of project areas and required level of detail appear to make it suitable for UAV image acquisition. However, the limited extent covered by the current UAV platform makes it less suitable for tasks which require covering large areas in a limited amount of time. Such applications could consider obtaining a UAV platform more suitable for the task in question, or smart sampling strategies.

The potential use of the DSM obtained from the imagery should also be further investigated. Comparing the quality of DSMs extracted from UAV imagery to traditional surveying methods and LiDAR is a being researched (e.g. Harwin and Lucieer, 2012, Haarbrink and Eisenbeiss, 2008). If the quality is sufficient, it could provide an enormous benefit to the upgrading project in the terms of surface water drainage analyses and the detailed design of infrastructure.

Currently the utility and adoption of UAVs for upgrading projects is limited by external factors. UAVs are an emerging technology, which are increasingly being used in developing countries for applications such as flood resilience in the Dar Ramani Huria project (http://ramanihuria.org/) and cadaster in the its4land project (http://www.its4land.com/) as well as unplanned settlement mapping. Unfortunately, legislation and protocols 
to obtain flight permission are often cumbersome or ambiguous. To resolve this issue, it is important to develop clear policies, guidelines and standards regarding flight regulations. In the case of Rwanda, legislation regarding UAV flight permissions is now implemented through the ministral regulations $\mathrm{N}^{\circ} 01 / \mathrm{MOS} / \mathrm{Trans} / 016$ of 26/04/2016 relating to unmanned civil aircraft systems. One of the objectives of the its4land project is also to identify current UAV flight regulations in East Africa and provide guidelines for their future development. On the other hand, the UAV pilots should operate safely and in compliance with these standards to help establish trust and transparency between the various parties. This could ensure UAV users have the freedom to conduct flights and extract high-quality information to support e.g. urban planning activities, while ensuring responsible flights and respecting public safety.

\section{V.Conclusions and recommendations}

To conclude, a UAV has the potential to be a valuable tool in spatial information acquisition for urban upgrading projects. The provision of highly-accurate and up-to-date information allows for the mapping of the current situation in the area, including the identification of buildings, roads, land use, drainage, and other points of interest which are vital for upgrading projects. Using UAV imagery provides advantages on four fronts. First, it allows more accurate information to be extracted by replacing conventional aerial or satellite imagery in existing project workflows. Second, UAV data products also have the potential to provide spatial information to the upgrading project which is not available through conventional image sources, such as providing high-resolution elevation information for detailed drainage calculations and the design of implementation measures. Third, the increased detail of the UAV imagery versus conventional satellite (or aerial) imagery saves time in field verification. Finally, the UAV images are also intuitively understandable by various project stakeholders, thus forming a foundation for effective communication of issues in the study area and planned interventions. Further analysis should investigate methods to obtain useful spatial information (i.e. semi-automatic classifications to identify different types of objects and their semantic attributes) from the data, which fit the needs of the stakeholders while taking local constraints into account.

To maximally benefit from the potential advantages of UAVs as a data acquisition platform for upgrading 
projects, the practical aspects of UAV data collection must be addressed. The spatial data requirements should be analyzed to enable the selection of a UAV platform, photogrammetric software, and hardware which is suited to the task at hand. Furthermore, effort should be made to explicitly consider the ethical issues involved with obtaining such high-resolution imagery over these impoverished and marginalized areas, and to stress the importance of sharing the benefits of the information obtained through the flights with the local population.

\section{VI.Acknowledgments}

The authors would like express their sincerest gratitude to the City of Kigali, CoK-OSC, RNRA, RHA, RCAA, village and sector officials, GeoTech, Voyants Solutions Pvt. Ltd., SG consultants, and other institutions for their collaboration and support during the UAV flights and for contributing through interviews.

\section{REFERENCES}

Abbott, J. 2002. A method-based planning framework for informal settlement upgrading. Habitat International, 26, 317-333.

Abbott, J. 2003. The use of GIS in informal settlement upgrading: its role and impact on the community and on local government. Habitat International, 27, 575-593.

Baiocchi, V., Dominici, D., Milone, M. V. \& Mormile, M. 2014. Development of a software to optimize and plan the acquisitions from UAV and a first application in a post-seismic environment. European Journal of Remote Sensing, 47, 477-496.

Banes, C. 2015. Upgrading of Unplanned Settlements in Urban Areas of Rwandan Cities - Guidance Note for Identification, Survey, Planning, Design, Implementation, Monitoring, Maintenance and Project Management. Kigali, Rwanda.

Barry, M. \& Rüther, H. 2005. Data collection techniques for informal settlement upgrades in Cape Town, South Africa. URISA Journal, 17, 43-52. 
Baud, I., Scott, D., Pfeffer, K., Sydenstricker-Neto, J. \& Denis, E. 2014. Digital and spatial knowledge management in urban governance: Emerging issues in India, Brazil, South Africa, and Peru. Habitat International, 44, 501-509.

Colomina, I. \& Molina, P. 2014. Unmanned aerial systems for photogrammetry and remote sensing: A review. ISPRS Journal of Photogrammetry and Remote Sensing, 92, 79-97.

Consortium, R. P. 2015. Rapid Planning - Sustainable Infrastructure, Environmental, and Resource Management for Highly Dynamic Metropolises. In: Steinbach, D., Schultheis, A., Lange, S., Peterek, M., Reichhardt, U., Hebbo, Y. \& Korovina, O. (eds.). Rapid Planning Consortium.

Davidson, F. \& Payne, G. 1983. Urban Projects Manual, Liverpool, Liverpool University Press.

Gistech Consultants Ltd 2015. Project Brief for Upgrading of Informal Settlement in Agatare Cell / Nyarugenge Sector. Kigali.

Goicoechea, A. 2008. Peparing Surveys for Urban Upgrading Interventions - Prototype Survey Instrument and User Guide. Urban Paper Series - UP 6. Washington D.C.: The World Bank.

Haarbrink, R. \& Eisenbeiss, H. 2008. Accurate DSM production from unmanned helicopter systems. International Archives of Photogrammetry, Remote Sensing and Spatial Information Sciences, 37, $1259-1264$.

Hartley, R. \& Zisserman, A. 2003. Multiple view geometry in computer vision, Cambridge university press. Harwin, S. \& Lucieer, A. 2012. Assessing the Accuracy of Georeferenced Point Clouds Produced via MultiView Stereopsis from Unmanned Aerial Vehicle (UAV) Imagery. Remote Sensing, 4, 1573-1599.

Kohli, D., Warwadekar, P., Kerle, N., Sliuzas, R. \& Stein, A. 2013. Transferability of Object-Oriented Image Analysis Methods for Slum Identification. Remote Sensing, 5, 4209-4228.

Kuffer, M., Barros, J. \& Sliuzas, R. V. 2014. The development of a morphological unplanned settlement index using very-high-resolution (VHR) imagery. Computers, Environment and Urban Systems, 48, 138152.

Mason, S. \& Fraser, C. 1998. Image sources for informal settlement management. The Photogrammetric Record, 16, 313-330. 
Meesuk, V., Vojinovic, Z., Mynett, A. E. \& Abdullah, A. F. 2015. Urban flood modelling combining top-view LiDAR data with ground-view SfM observations. Advances in Water Resources, 75, 105-117.

Nebiker, S., Annen, A., Scherrer, M. \& Oesch, D. 2008. A light-weight multispectral sensor for micro UAVOpportunities for very high resolution airborne remote sensing. The international archives of the photogrammetry, remote sensing and spatial information sciences, 37, 1193-1200.

Nex, F. \& Remondino, F. 2014. UAV for 3D mapping applications: a review. Applied Geomatics, 6, 1-15.

Nlesc. 2015. Processing large datasets on consumer-grade computers [Online]. Netherlands eScience Center. Available: $\quad$ https://www.esciencecenter.nl/project/improving-open-source-photogrammetricworkflows-for-processing-big-datasets [Accessed 12-01 2016].

Paar, P. \& Rekittke, J. 2011. Low-Cost Mapping and Publishing Methods for Landscape Architectural Analysis and Design in Slum-Upgrading Projects. Future Internet, 3, 228-247.

Pannell, D. J., Marshall, G. R., Barr, N., Curtis, A., Vanclay, F. \& Wilkinson, R. 2011. Understanding and promoting adoption of conservation practices by rural landholders. Changing Land Management: Adoption of New Practices by Rural Landholders, 11.

Pfeffer, K., Baud, I., Denis, E., Scott, D. \& Sydenstricker-Neto, J. 2013. Participatory Spatial Knowledge Management Tools: Empowerment and upscaling or exclusion? Information, Communication \& Society, 16, 258-285.

Rambaldi, G., Chambers, R., Mccall, M. \& Fox, J. 2006. Practical ethics for PGIS practitioners, facilitators, technology intermediaries and researchers. Participatory Learning and Action, 54, 106-113.

Remondino, F., Spera, M. G., Nocerino, E., Menna, F. \& Nex, F. 2014. State of the art in high density image matching. The Photogrammetric Record, 29, 144-166.

Rwanda, G. O. 2000. Rwanda Vision 2020. Kigali: Ministry of Finance and Economic Planning.

Sona, G., Pinto, L., Pagliari, D., Passoni, D. \& Gini, R. 2014. Experimental analysis of different software packages for orientation and digital surface modelling from UAV images. Earth Science Informatics, 7, 97-107. 
Taubenböck, H. \& Kraff, N. J. 2013. The physical face of slums: a structural comparison of slums in Mumbai, India, based on remotely sensed data. Journal of Housing and the Built Environment, 29, 15-38.

UN-Habitat 2012. Streets as tools for urban transformation in slums: A Street-led Approach to Citywide Slum Upgrading. Nairobi. 